\title{
Multi-Stage Prediction of Feed System Time Series
}

\author{
Lihua SHEN ${ }^{\mathrm{a}}$, Biling WANG ${ }^{\mathrm{b} 1}$, Hongjun $\mathrm{LIU}^{\mathrm{a}}$ \\ a Shenyang Aerospace University, China \\ ${ }^{\mathrm{b}}$ Avic Aerodynamics Research Institute of China, China
}

\begin{abstract}
In order to reduce the tracking error of the computer numerical control (CNC) feed system and improve the CNC machining accuracy, a novel prediction model is proposed based on fuzzy C-means robust variational echo state network. Firstly, the feed speed time series is clustered, and then reconstructed for different categories. The multi-stage robust prediction models are established to realize the multi-state robust prediction of the $\mathrm{CNC}$ machining feed velocity to reduce the tracking error of the feed system. Finally, the reference and actual time series with different feed speed are used to verify the established models. The results show that the proposed method can reduce the tracking error and realize the effective prediction of the time series of the feed system.
\end{abstract}

Keywords. CNC, multi-stage, cluster, robust prediction, time series

\section{Introduction}

The modeling and prediction methods which are often used for error compensation include physical model driven prediction methods and data driven prediction methods. The physical model-driven forecasting methods need the specific structure of the physical model and corresponding parameters to be set in advance, while it is always difficult for complex control systems to establish accurate physical models. Consequently, physical model driven methods such as iterative control method [1] and its improvement [2] are subject to many restrictions in practical applications. The other type of methods to solve the above problems are the data-driven prediction methods. Two typical and commonly used error modeling prediction methods in this type of method are the neural network prediction method [3-5] and the support vector machine for regression (SVR) prediction method [6]. However, the error modeling based on wavelet neural network [3] and LSTM [4] is time-consuming and the BP [5] method is not robust. The SVR in [6] just predict time series without establishing specific models for different feed states.

As a data-driven model, neural network has strong data modeling capabilities and nonlinear approximation capabilities. Especially the robust neural network methods have found an increasingly wide utilization in time series prediction [7,8]. Compared with the traditional neural network, the echo state network (ESN) [9] has the advantages of fast training speed and considering the temporal correlation of data. It

1 Corresponding author: Avic Aerodynamics Research Institute of China, China; E-mail: sacslh@126.com. 
has been more and more used in complex system modeling and time series prediction, and has achieved good prediction results. And its improvement robust variational echo state network (RVESN) [10] is proved to be robust for data analysis with noise and outliers. In view of the characteristics of the time series of the feed system and the modeling ability of the RVESN in the time series prediction, a multi-stage feed system time series prediction method based on the fuzzy C-means (FCM) [11] and the RVESN named FCM-RVESN is proposed. Firstly, the first-order difference of the reference speed is extracted, and then FCM is used to cluster the data into stable processing state and acceleration-deceleration state. Subsequently two clusters are implemented phase space reconstruction respectively, and prediction models for the reconstructed time series are established based on RVESN. The rest of this paper is structured as follows. Section 2 gives a brief review of the preliminary works. Section 3 presents the proposed FCM-RVESN model. Section 4 gives the simulation results of time series from circle and linear motions of practical CNC feed system. In Section 5, the conclusions are given.

\section{Robust Echo State Network}

The ESN [9] always has a large reservoir to map data to higher-dimensional space. It often discards the initial transient samples in the prediction process to improve the accuracy of the network prediction. When the length of the transient state is $l-1$, suppose the state matrix of the reservoir and the target output matrix are $\mathbf{A}$ and $\mathbf{y}$ respectively. For the convenience of subsequent calculations, each row of

$\mathbf{A}$ is set as $\mathbf{a}_{k}$, and assume that $N$ samples are retained, $\mathbf{A} \in R^{N \times r}$ then:

$$
\begin{aligned}
\mathbf{A} & =[x(l), x(l+1), x(l+2), \ldots, x(l+N-1)]^{T} \\
\mathbf{y} & =[y(l), y(l+1), y(l+2), \ldots y(l+N-1)]^{T}
\end{aligned}
$$

According to the echo state network structure, Eq. (3) is obtained:

$$
\mathbf{A w}=\mathbf{y}
$$

The RVESN uses the robust Gaussian mixture distribution as the model output likelihood function. For any training sample, the formula is as follows:

$$
p(y(k))=\eta p(y(k))+(1-\eta) p_{0}(y(k))
$$

Among them, $\eta$ is an adaptive parameter that is automatically tuned as the Outliers and noise number and proportion of data set. $p(\mathbf{y})$ and $p_{0}(\mathbf{y})$ are as follows:

$$
\begin{gathered}
p(\mathbf{y} \mid \mathbf{w}, \beta)=\left(\frac{\beta}{2 \pi}\right)^{N / 2} \exp \left\{-\frac{\beta}{2}\|\mathbf{y}-\mathbf{A} \mathbf{w}\|^{2}\right\} \\
p_{0}(\mathbf{y})=\left(\frac{\beta_{0}}{2 \pi}\right)^{N / 2} \exp \left\{-\frac{\beta_{0}}{2}\|\mathbf{y}-\mathbf{A w}\|^{2}\right\}
\end{gathered}
$$

The approximate posterior probability distribution of $\mathbf{w}$ is Gaussian, and the covariance matrix and mean value are $\Sigma$ and $\boldsymbol{\mu}$ respectively: 


$$
\begin{gathered}
\sum=\left(\sum_{k=1}^{N}\left[\beta_{0}\left(1-E_{z}\left(z_{k}\right)\right)+\beta E_{z}\left(z_{k}\right)\right] \mathbf{a}_{k}^{T} \mathbf{a}_{k}+\operatorname{diag}\left(\alpha_{h}\right)\right)^{-1} \\
\boldsymbol{\mu}=\sum\left(\sum_{k=1}^{N}\left[\beta_{0}\left(1-E_{z}\left(z_{k}\right)\right)+\beta E_{z}\left(z_{k}\right)\right] y(k) \mathbf{a}_{k}^{T}\right)
\end{gathered}
$$

\section{FCM-RVESN}

\subsection{Feature Extraction and State Clustering}

Through the analysis of the time series for linear and circle motions of the feed system, it is found that the first-order difference of the data is significantly different in the stable state and the acceleration-deceleration state. Therefore, features of the data at different feed speed are extracted by the first-order difference, and then FCM is used to cluster the time series based on the extraction results.

Suppose the time series generated at a certain feed rate has $N$ samples with $d$ dimensions, which is expressed as $\mathbf{x}(i)=\left[x_{1}(i), x_{2}(i), \ldots, x_{d}(i)\right]^{\mathrm{T}} \in R^{d}, N-1$ samples with $d$ dimensions are obtained by implementing first difference.

After the above-mentioned first-order difference, FCM is utilized to cluster absolute value of the above data into two states. The time series to be clustered can be expressed as follows:

$$
\begin{aligned}
& x_{f c m}(i)=a b s\left[x_{1}(i+1)-x_{1}(i)\right] \\
& x_{f c m}(i+1)=a b s\left[x_{1}(i+2)-x_{1}(i+1)\right] \\
& \cdots \cdots \\
& x_{f c m}(N-1)=a b s\left[x_{1}(N)-x_{1}(N-1)\right]
\end{aligned}
$$

The FCM [11] defines the objective function to be optimized :

$$
J m=\sum_{i=1}^{N} \sum_{j=1}^{C} u_{i j}^{m}\left(d_{i j}\right)^{2}
$$

In the Eq. (10), $m$ is Weighted index, and $d_{i j}=\left\|x_{i}-c_{j}\right\|^{2}$ is the distance between the ith time series and the centre of the $j$ th cluster, which is stable state or acceleration-deceleration state.In the objective function, $C$ is number of the clusters, which is equal to two. $N$ is the number of samples, $u_{i j}$ is Membership degree that $i$ belongs to the $j t h$ cluster, $x_{i}$ is the ith sample, and $c_{j}$ is the centre of the $j t h$ cluster. Finally, the speed time series are divided into two clusters: stable state and acceleration-deceleration state. 


\subsection{FCM-RVESN Prediction Model}

Due to the characteristics of the time series, the actual speed is not only related to the reference speed at the current moment, but also related to the reference speed and actual speed at the previous moments. Therefore, it needs to be reconstructed in phase space. According to Takens theorem, as long as a suitable embedding dimension is found, the original time series can be reconstructed to obtain a dynamic system with a higher dimension than the input variable, and its prediction accuracy can be improved by reconstructing the time series.

After first-order difference and phase space reconstruction of the time series, the FCM-RVESN feed system time series prediction models of two states are established at different speed and the model structure is as figure 1:

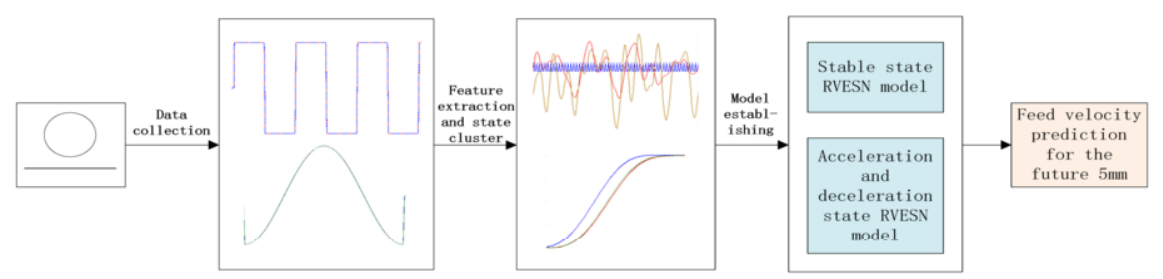

Figure 1. Structure of FCM-RVESN.

First, the reference speed and the actual speed of the semi-closed loop on each coordinate axis are collected at different feed speed in the process of linear and circular interpolation. The first-order difference is performed on the collected data to extract features, and then FCM is used to cluster the collected data. The time series in the stable state and the acceleration-deceleration state are obtained. The time series is reconstructed in different time processes and different states respectively. Subsequently, RVESN models for the reconstructed time series are established in different states.

\section{Experimental Results}

In the feed servo drive system, the excitation signal is set as $x(t)$, which is also the input reference speed, and $y(t)$ is the semi-closed loop actual response signal, which is the actual speed of the servo motor encoder. In the semi-closed loop speed prediction of the feed system, the previous $x(t)$ and $y(t)$ are used to predict $y(t+\delta)$. The value of the prediction horizon $\delta$ is set according to actual CNC system. For single-step prediction, $\delta$ is equal to 1 . In each simulation experiment of this paper, $\delta$ is set as 5 , which means that the actual speed of the fifth millisecond in the future is predicted. The reason why it is set to 5 is because the delay time of the servo is about 3 to 4 milliseconds between received the reference data and outputting response signal.The data used in this chapter is generated by linear and circular motions. Parameters used in the experiments are as follows. Data generated by the two motions will be analyzed respectively. 
Table 1. parameters of the experiments

\begin{tabular}{|c|c|c|c|c|c|}
\hline $\begin{array}{l}\text { Size of } \\
\text { reservoir }\end{array}$ & Sparseness & $\begin{array}{l}\text { Spectral } \\
\text { Radius }\end{array}$ & $\begin{array}{c}\text { Regularization } \\
\text { Coefficient of SVESN }\end{array}$ & $\begin{array}{l}\text { Cluster } \\
\text { Number }\end{array}$ & $\begin{array}{l}\text { Prediction } \\
\text { Horizon }\end{array}$ \\
\hline 100 & 0.05 & 0.95 & 2 & 2 & 5 \\
\hline
\end{tabular}

\subsection{Circle Time Series}

The two-axis arc motion path is a circle with diameter of $100 \mathrm{~mm}$. By setting different feed speed as $4000 \mathrm{~mm} / \mathrm{min}$ and $5000 \mathrm{~mm} / \mathrm{min}$, the time series at different preset feed speed can be obtained. In the case of circular motion. After decomposing the composite speed to the $\mathrm{X}$-axis and $\mathrm{Y}$-axis, the speed on the $\mathrm{X}$-axis is generally a sine curve, and the speed on the Y-axis is generally a cosine curve. Parameters are set as table 1 . The speed prediction results on $\mathrm{X}$-axis are shown in the table 2 and figure 2, which demonstrate that the SVR has higher precision while predicting feed speed at $5000 \mathrm{~mm} / \mathrm{min}$, but FCM-RVESN has higher precision compared with SVR, SVESN and actual system accuracy while predicting feed speed at $4000 \mathrm{~mm} / \mathrm{min}$.

Table 2. comparison of prediction results (circle time series).

\begin{tabular}{ccccc}
\hline $\begin{array}{c}\text { Feed } \\
\text { speed }\end{array}$ & $\begin{array}{c}\text { Actual } \\
\text { error }\end{array}$ & SVESN & SVR & FCM-RV \\
\hline 4000 & 16.3927 & 14.2434 & 3.2185 & ESN \\
\hline 5000 & 23.8857 & 21.2286 & $\mathbf{1 . 5 5 4 9}$ & 1.9804 \\
\hline
\end{tabular}
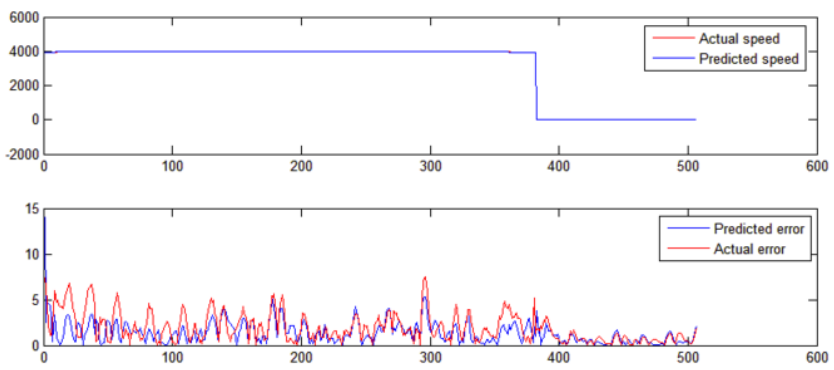

Figure 2. Prediction results of circle (stable state at $4000 \mathrm{~mm} / \mathrm{min}$ )

\subsection{Linear Time Series}

The single-axis linear motion path length is $100 \mathrm{~mm}$. By setting feed speed as $4000 \mathrm{~mm} / \mathrm{min}$ and $5000 \mathrm{~mm} / \mathrm{min}$, the time series at different preset feed speed can be obtained. The speed prediction results are shown in the table 3 and figure 3 , which demonstrate that the FCM-RVESN has higher precision compared with SVR, SVESN and actual system accuracy in two state and it is a feasible model for the speed prediction of CNC feed system. 
Table 3. comparison of prediction results (linear time series).

\begin{tabular}{ccccc}
\hline Feed speed & Actual error & SVESN & SVR & FCM-RVESN \\
\hline 4000 & 133.1942 & 2.8139 & 2.2177 & $\mathbf{1 . 9 6 2 7}$ \\
5000 & 175.9164 & 3.1871 & 2.5911 & $\mathbf{2 . 3 4 1 4}$ \\
\hline
\end{tabular}
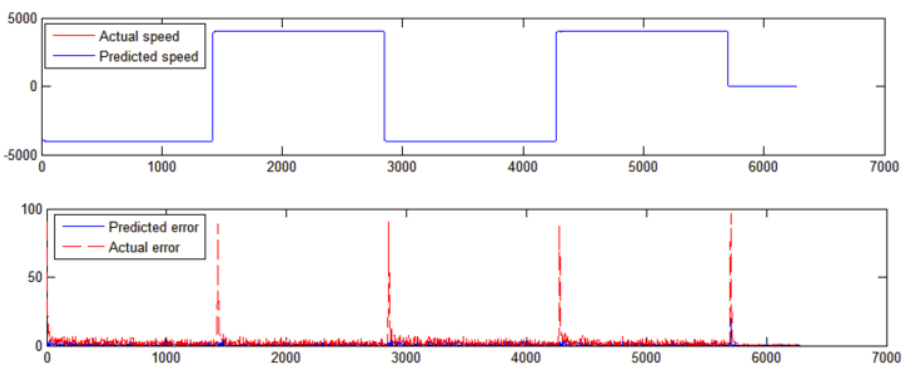

Figure 3. Prediction results of linear time series (acceleration-deceleration state at $4000 \mathrm{~mm} / \mathrm{min}$ )

\section{Conclusion}

In this paper, the time series from the feed system of CNC machine tools is analyzed, clustered and a multi-stale FCM-RVESN prediction method is proposed at different feed speed. The actual speed of practical CNC feed system is forecasted, and the prediction results are compared with SVR, SVESN and actual measured error. It is found that the proposed method has higher precision in three datasets for all the datasets used in the experiments.

\section{Acknowledgements}

The authors would like to thank the financial support from the PhD Start-up Fund of Shenyang Aerospace University (120419026/502) and the fund of "Research on load prediction method of wind Tunnel balance calibration based on intelligent algorithm".

\section{References}

[1] Ahn HS, Chen YQ, Moore KL. Iterative learning control: brief survey and categorization. IEEE Transactions on Systems Man \& Cybernetics Part C, 2007, 37(6): 1099-1121

[2] Tsai MS, Yen CL, Yau HT. Integration of an empirical mode decomposition algorithm with iterative learning control for high-precision machining. IEEE/ASME Transactions on Mechatronics, 2013, 18(3): $878-886$

[3] Ustundag BB, Kulaglic A. High-performance time series prediction with predictive error compensated wavelet neural networks. IEEE Access, 2020, 8: 210532-210541.

[4] Jiang Y, Chen J, Zhou H et al. Nonlinear time-series modeling of feed drive system based on motion states classification. Journal of Intelligent Manufacturing, 2020, 31(8).

[5] Yin Q, Tan F, Chen H et al. Spindle thermal error modeling based on selective ensemble BP neural networks. The International Journal of Advanced Manufacturing Technology, 2019, 101(5): 1699-1713. 
[6] Lei M, Jiang G, Yang J et al. Thermal error modeling with dirty and small training sample for the motorized spindle of a precision boring machine. The International Journal of Advanced Manufacturing Technology, 2017, 93(1): 571-586.

[7] Li D, Han M, Wang J. Chaotic time series prediction based on a novel robust echo state network. IEEE Transactions on Neural Networks and Learning Systems, 2012, 23(5): 787-799

[8] Altan A, Karasu S. The effect of kernel values in support vector machine to forecasting performance of financial time series. The Journal of Cognitive Systems, 2019, 4(1): 17-21.

[9] Jaeger H, Haas H. Harnessing nonlinearity: Predicting chaotic systems and saving energy in wireless communication. Science, 2004, 304(5667): 78-80

[10] Shen L, Chen J, Zeng Z et al. A novel echo state network for multivariate and nonlinear time series prediction. Applied Soft Computing, 2018, 62: 524-535.

[11] Ghosh S, Dubey SK. Comparative analysis of k-means and fuzzy c-means algorithms. International Journal of Advanced Computer Science and Applications, 2013, 4(4). 